\title{
Graphitized Biocarbon Derived from Hydrothermally Liquefied Low-Ash Corn Stover Supplementary Material
}

K.M. Shell ${ }^{1}$, V.S. Amar ${ }^{2}$, J.A. Bobb ${ }^{1}$, S. Hernandez ${ }^{3}$, R.V. Shende ${ }^{2}$, and Ram B. Gupta ${ }^{1 *}$

${ }^{1}$ Department of Chemical and Life Science Engineering, Virginia Commonwealth University, Richmond, Virginia 23284, USA.

${ }^{2}$ Chemical and Biological Engineering Department, South Dakota School of Mines \& Technology, Rapid City, South Dakota 57701, USA.

${ }^{3}$ Bioenergy Technologies Department, Clean Energy \& Transportation Division, Idaho National Laboratory, Idaho Falls, Idaho 83415, USA.

Supplemental Instruction prepared for possible publication with corresponding manuscript in Industrial Engineering and Chemistry Research

December 11, 2021

\section{Correspondence}

$1 *$ Ram B. Gupta, Department of Chemical and Life Science Engineering, Virginia Commonwealth University, Richmond, Virginia 23284 USA.

E-Mail: rbgupta@vcu.edu 


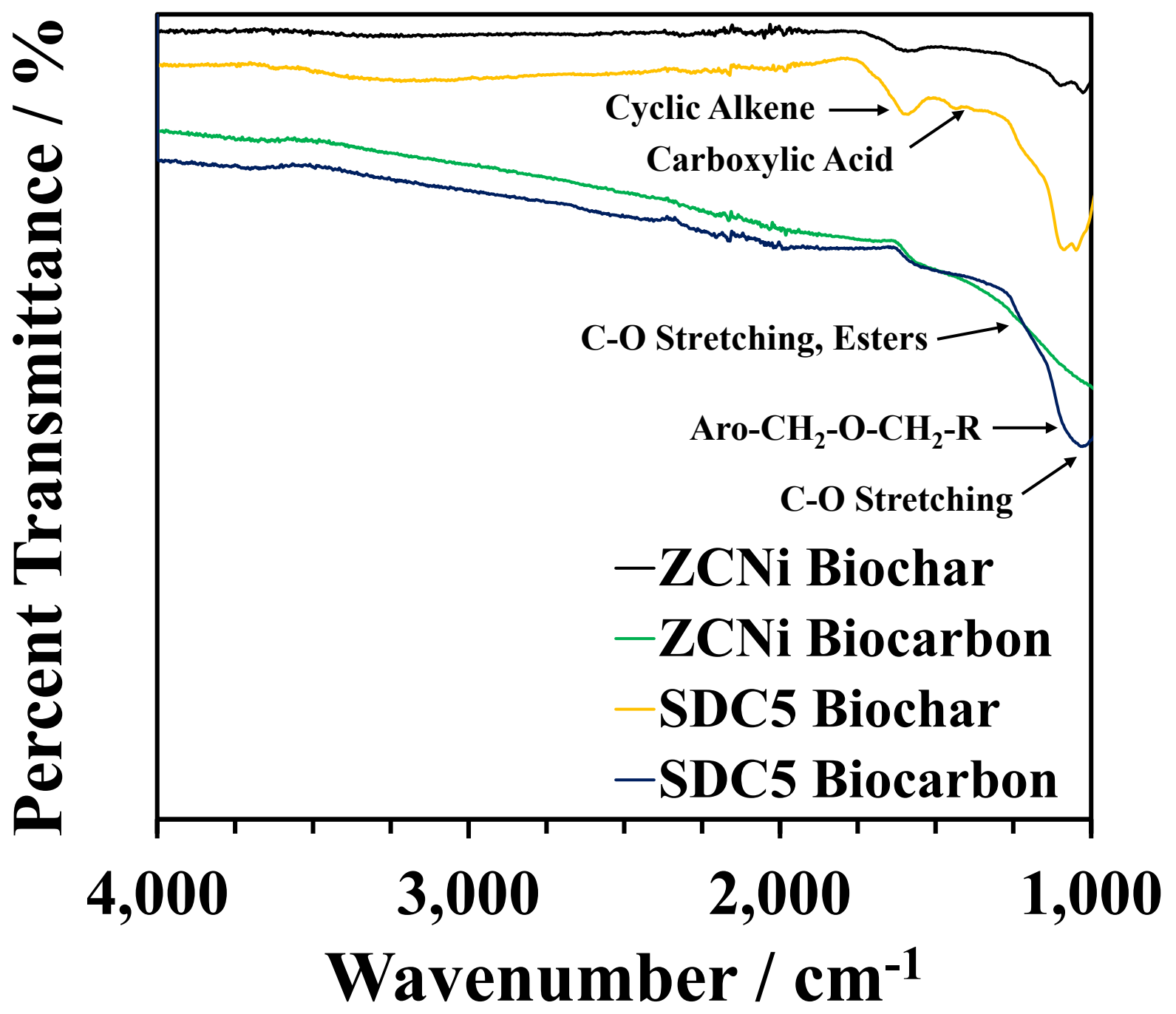

Figure S1. ATR-FTIR Spectrum denoting both SDC5 and ZCNi materials.

Table S1. Reported parameters of various biomass-derived biocarbons. 


\begin{tabular}{|c|c|c|c|c|c|c|c|c|}
\hline Biomass & $\begin{array}{l}\text { Activation } \\
\text { method }\end{array}$ & $\begin{array}{c}\text { SSA } \\
\left(\mathrm{m}^{2} \mathrm{~g}^{-1}\right)\end{array}$ & $\begin{array}{c}\mathrm{V}_{\mathrm{BET}} \\
\left(\mathrm{cm}^{3} \mathrm{~g}^{-1}\right)\end{array}$ & $\begin{array}{c}\text { Capacitance } \\
\left(\mathrm{F} \mathrm{g}^{-1}\right)\end{array}$ & $\begin{array}{c}\text { Retention } \\
(\%)\end{array}$ & $\begin{array}{l}\text { Reported } \\
\text { Cycles }\end{array}$ & Electrolyte & Ref. \\
\hline $\begin{array}{l}\text { Corn } \\
\text { Stover }\end{array}$ & $\mathrm{ZnCl}_{2} / \mathrm{NiNO}_{3}$ & 753 & 0.27 & 316 & 100 & 10000 & $2 \mathrm{M} \mathrm{KOH}$ & - \\
\hline $\begin{array}{l}\text { Corn } \\
\text { Stover }\end{array}$ & $\begin{array}{l}\text { Microwave } \\
\text { pyrolysis }\end{array}$ & 1433 & 0.76 & 246 & $\mathrm{n} / \mathrm{a}$ & $\mathrm{n} / \mathrm{a}$ & $\mathrm{n} / \mathrm{a}$ & 2 \\
\hline $\begin{array}{l}\text { Corn } \\
\text { Stover }\end{array}$ & $\begin{array}{c}\text { Facile } \\
\text { thermal } \\
\text { carbonization }\end{array}$ & 215 & 0.12 & 242 & 92 & 2500 & $2 \mathrm{M} \mathrm{KOH}$ & 1 \\
\hline Corn Cob & $\mathrm{KOH}$ & 3054 & 1.50 & 328 & 91 & 10000 & $0.5 \mathrm{M} \mathrm{H}_{2} \mathrm{SO}_{4}$ & 3 \\
\hline Corn Stem & $\mathrm{KOH}$ & 1420 & $\mathrm{n} / \mathrm{a}$ & 232 & $\mathrm{n} / \mathrm{a}$ & 10000 & $\mathrm{n} / \mathrm{a}$ & 4 \\
\hline Corn Silk & $\mathrm{KOH}$ & 2285 & 1.44 & 160 & 87.6 & $\mathrm{n} / \mathrm{a}$ & $6 \mathrm{M} \mathrm{KOH}$ & 5 \\
\hline $\begin{array}{l}\text { Corn } \\
\text { Husk }\end{array}$ & $\begin{array}{c}\mathrm{KOH}, \\
\text { thermal } \\
\text { carbonization }\end{array}$ & 867 & 0.51 & 356 & 95 & 2500 & $6 \mathrm{M} \mathrm{KOH}$ & 6 \\
\hline Corn Stalk & $\begin{array}{c}\mathrm{KOH}, \mathrm{Ni} \\
\text { catalyst }\end{array}$ & 2495 & 1.23 & 323 & 98 & 1000 & $6 \mathrm{M} \mathrm{KOH}$ & 7 \\
\hline Corn Stalk & $\begin{array}{c}\text { Fe catalyst, } \\
\text { pyrolysis }\end{array}$ & 540 & 0.48 & 213 & $\begin{array}{c}99 \\
\text { (columb.) }\end{array}$ & 6000 & $6 \mathrm{M} \mathrm{KOH}$ & 8 \\
\hline $\begin{array}{l}\text { Corn } \\
\text { Straw }\end{array}$ & $\begin{array}{l}\text { Hydrothermal } \\
\text { carbonization }\end{array}$ & 1771 & 1.85 & 222 & 94 & 5000 & $6 \mathrm{M} \mathrm{KOH}$ & 9 \\
\hline $\begin{array}{l}\text { Corn } \\
\text { Starch }\end{array}$ & $\mathrm{H}_{3} \mathrm{PO}_{4}$ & 1167 & 1.80 & 162 & 93 & 5000 & $6 \mathrm{M} \mathrm{KOH}$ & 10 \\
\hline Fungi & Hydrothermal & 80 & 0.50 & 196 & 99 & 1000 & $6 \mathrm{M} \mathrm{KOH}$ & 11 \\
\hline Fish scale & $\mathrm{KOH}$ & 2273 & 2.74 & 168 & 77 & $\mathrm{n} / \mathrm{a}$ & $7 \mathrm{M} \mathrm{KOH}$ & 12 \\
\hline Seaweed & Thermal & $\begin{array}{c}15- \\
1307\end{array}$ & $\mathrm{n} / \mathrm{a}$ & 119 264 & 89 & 10000 & $1 \mathrm{M} \mathrm{H}_{2} \mathrm{SO}_{4}$ & 13 \\
\hline $\begin{array}{l}\text { Walnut } \\
\text { shell }\end{array}$ & $\mathrm{KOH}$ & $\begin{array}{c}2044- \\
2390\end{array}$ & $\begin{array}{c}0.24- \\
1.03\end{array}$ & $168-203$ & $67-87$ & 1000 & $3 \mathrm{M} \mathrm{H}_{2} \mathrm{SO}_{4}$ & 14 \\
\hline $\begin{array}{l}\text { Tea- } \\
\text { leaves }\end{array}$ & $\mathrm{KOH}$ & $\begin{array}{l}2245- \\
2841\end{array}$ & $\begin{array}{c}1.07- \\
1.37\end{array}$ & $275-330$ & 92 & 2000 & $2 \mathrm{M} \mathrm{KOH}$ & 15 \\
\hline $\begin{array}{l}\text { Waste } \\
\text { paper }\end{array}$ & $\mathrm{KOH}$ & 416 & 0.225 & 180 & 99 & 2300 & $6 \mathrm{M} \mathrm{KOH}$ & 16 \\
\hline
\end{tabular}




\begin{tabular}{|c|c|c|c|c|c|c|c|c|}
\hline $\begin{array}{l}\text { Banana } \\
\text { peel }\end{array}$ & Hydrothermal & 1650 & 1.26 & 206 & 88 & 1000 & $6 \mathrm{M} \mathrm{KOH}$ & 17 \\
\hline $\begin{array}{l}\text { Sunflower } \\
\text { seed shell }\end{array}$ & $\mathrm{KOH}$ & $\begin{array}{l}619- \\
2585\end{array}$ & $\begin{array}{c}0.48- \\
0.62\end{array}$ & $213-311$ & $\mathrm{n} / \mathrm{a}$ & $\mathrm{n} / \mathrm{a}$ & $30 \% \mathrm{KOH}$ & 18 \\
\hline $\begin{array}{l}\text { Wheat } \\
\text { straw }\end{array}$ & $\mathrm{KOH}$ & 2316 & 1.50 & 251 & $\mathrm{n} / \mathrm{a}$ & $\mathrm{n} / \mathrm{a}$ & $\mathrm{MeEt}_{3} \mathrm{NBF}_{4} / \mathrm{AN}$ & 19 \\
\hline \multirow{2}{*}{$\begin{array}{l}\text { Silk } \\
\text { protein }\end{array}$} & \multirow{2}{*}{$\mathrm{KOH}$} & \multirow{2}{*}{2557} & \multirow{2}{*}{$\mathrm{n} / \mathrm{a}$} & 264 & 93 & \multirow{2}{*}{10000} & $1 \mathrm{M} \mathrm{H}_{2} \mathrm{SO}_{4}$ & 20 \\
\hline & & & & 168 & 97 & & $\mathrm{BMIM} \mathrm{BF} / \mathrm{AN}$ & \\
\hline \multirow{2}{*}{ Pollen } & \multirow{2}{*}{$\begin{array}{l}\text { Hydrothermal } \\
\text { and } \mathrm{KOH}\end{array}$} & \multirow{2}{*}{3037} & \multirow{2}{*}{2.27} & & & \multirow{2}{*}{5000} & $\mathrm{TEABF}_{4} / \mathrm{AN}$ & \\
\hline & & & & 207 & 95 & & $\mathrm{EMIM} \mathrm{BF}_{4}$ & 21 \\
\hline Rice husk & $\mathrm{CO}_{2}$ & 1500 & $\mathrm{n} / \mathrm{a}$ & 76 & 95 & 5000 & $\mathrm{TEABF}_{4} / \mathrm{PC}$ & 22 \\
\hline $\begin{array}{l}\text { Animal } \\
\text { bone }\end{array}$ & $\mathrm{KOH}$ & 2157 & 2.26 & 185 & $\mathrm{n} / \mathrm{a}$ & $\mathrm{n} / \mathrm{a}$ & $7 \mathrm{M} \mathrm{KOH}$ & 23 \\
\hline \multirow{2}{*}{$\begin{array}{l}\text { Cherry } \\
\text { stone }\end{array}$} & \multirow{2}{*}{$\mathrm{KOH}$} & \multirow{2}{*}{1171} & \multirow{2}{*}{0.67} & 232 & \multirow{2}{*}{$\mathrm{n} / \mathrm{a}$} & \multirow{2}{*}{$\mathrm{n} / \mathrm{a}$} & $2 \mathrm{M} \mathrm{H}_{2} \mathrm{SO}_{4}$ & \multirow[t]{2}{*}{24} \\
\hline & & & & 120 & & & $\mathrm{TEABF}_{4} / \mathrm{AN}$ & \\
\hline \multirow{2}{*}{ Fir wood } & $\mathrm{KOH}$ & 1064 & 0.61 & 180 & \multirow{2}{*}{$\mathrm{n} / \mathrm{a}$} & \multirow{2}{*}{$\mathrm{n} / \mathrm{a}$} & \multirow{2}{*}{$0.5 \mathrm{M} \mathrm{H}_{2} \mathrm{SO}_{4}$} & 25 \\
\hline & Steam & 1016 & 0.75 & 110 & & & & \\
\hline \multirow{2}{*}{$\begin{array}{l}\text { Coconut } \\
\text { shell }\end{array}$} & \multirow{2}{*}{$\mathrm{ZnCl}_{2}$} & \multirow{2}{*}{1874} & \multirow{2}{*}{1.21} & 268 & $>99$ & \multirow{2}{*}{5000} & $6 \mathrm{M} \mathrm{KOH}$ & \multirow[t]{2}{*}{26} \\
\hline & & & & 196 & $\mathrm{n} / \mathrm{a}$ & & $\mathrm{TEABF}_{4} / \mathrm{PC}$ & \\
\hline Lignin & $\mathrm{KOH}$ & 3775 & 2.70 & 286.7 & $\mathrm{n} / \mathrm{a}$ & $\mathrm{n} / \mathrm{a}$ & $6 \mathrm{M} \mathrm{KOH}$ & 27 \\
\hline Lignin & $\mathrm{KOH}$ & 2265 & $\mathrm{n} / \mathrm{a}$ & 336 & 100 & 1000 & $6 \mathrm{M} \mathrm{KOH}$ & 28 \\
\hline Lignin & Composite & 802 & $\mathrm{n} / \mathrm{a}$ & 880 & 96 & 5000 & $6 \mathrm{M} \mathrm{KOH}$ & 29 \\
\hline Lignin & $\mathrm{KOH}$ & 2957 & 1.79 & 348 & 100 & 10000 & $1 \mathrm{M} \mathrm{KOH}$ & 30 \\
\hline
\end{tabular}




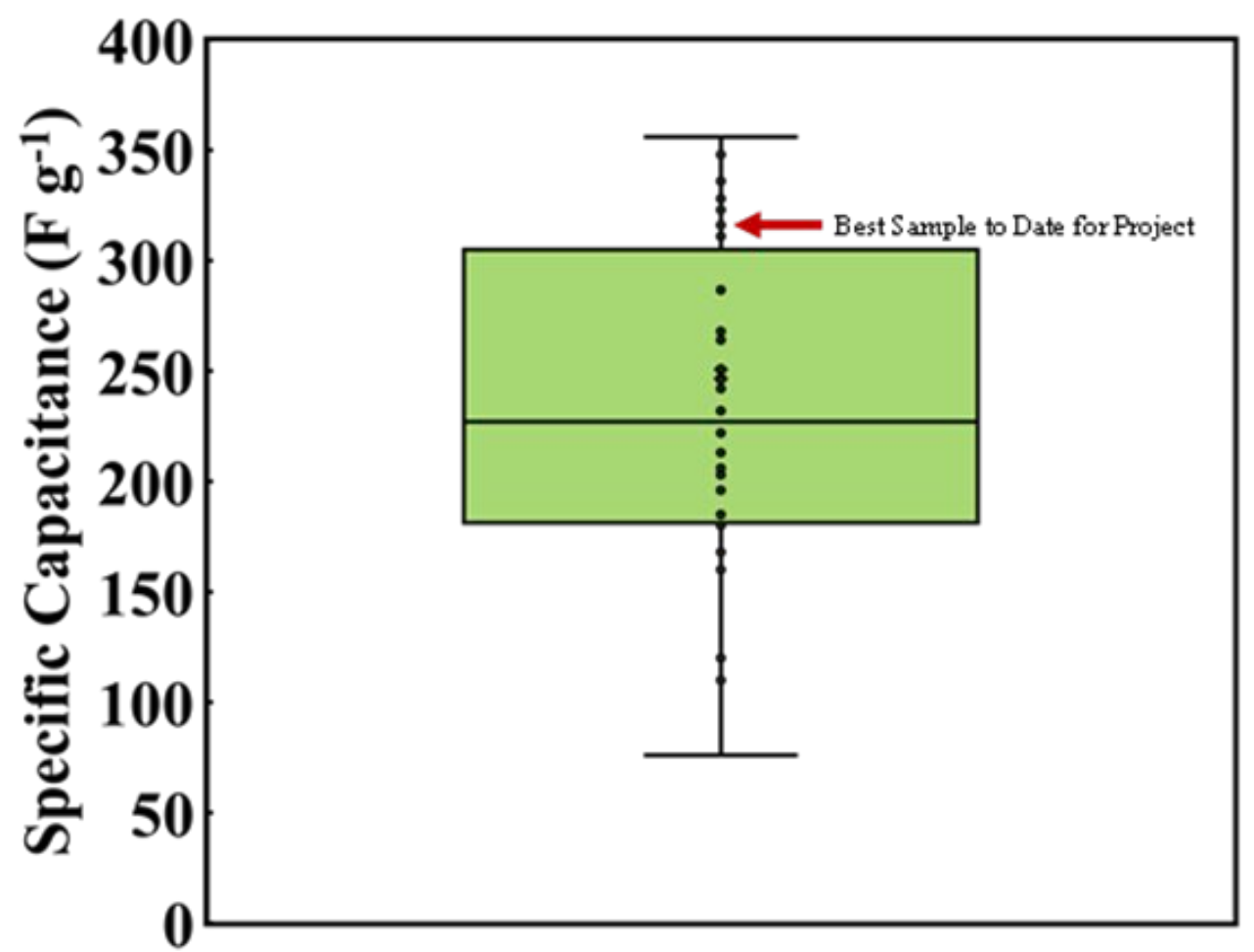

Figure S2. Box and whisker plot of specific capacitance values denoted in Table S1.

\section{References}

1. Shell, K. M., Rodene, D. D., Amar, V., Thakkar, A., Maddipudi, B., Kumar, S., ... \& Gupta, R. B. (2021). Supercapacitor performance of corn stover-derived biocarbon produced from the solid co-products of a hydrothermal liquefaction process. Bioresource Technology Reports, 13, 100625.

2. Jin, H., Wang, X., Shen, Y., Gu, Z., 2014. A high-performance carbon derived from corn stover via microwave and slow pyrolysis for supercapacitors. J. Anal. Appl. Pyrolysis 110, 18-23. https://doi.org/10.1016/j.jaap.2014.07.010.

3. Wang, D., Geng, Z., Li, B., \& Zhang, C. (2015). High performance electrode materials for electric double-layer capacitors based on biomass-derived activated carbons. Electrochimica Acta, 173, 377-384.

4. Lee, M.E., Choe, J.H., Yun, Y.S., Jin, H.-J., 2017. Corn stem-derived, hierarchically nanoporous carbon as electrode material for supercapacitors. Nanosci. Nanotechnol. 17, 7729-7734. https://doi.org/10.1166/jnn.2017.14773.

5. Mitravinda, T., Nanaji, K., Anandan, S., Jyothirmayi, A., Chakravadhanula, V. S. K., Sharma, C. S., \& Rao, T. N. (2018). Facile synthesis of corn silk derived nanoporous carbon for an improved supercapacitor performance. Journal of the electrochemical society, 165(14), A3369. 
6. Song, S., Ma, F., Wu, G., Ma, D., Geng, W., \& Wan, J. (2015). Facile self-templating large scale preparation of biomass-derived 3D hierarchical porous carbon for advanced supercapacitors. Journal of materials chemistry A, 3(35), 18154-18162.

7. Cao, Y., Wang, K., Wang, X., Gu, Z., Fan, Q., Gibbons, W., ... \& Shrestha, M. (2016). Hierarchical porous activated carbon for supercapacitor derived from corn stalk core by potassium hydroxide activation. Electrochimica Acta, 212, 839-847.

8. Wang, L., Mu, G., Tian, C., Sun, L., Zhou, W., Yu, P., ... \& Fu, H. (2013). Porous graphitic carbon nanosheets derived from cornstalk biomass for advanced supercapacitors. ChemSusChem, 6(5), 880-889.

9. Lu, Y., Zhang, S., Yin, J., Bai, C., Zhang, J., Li, Y., Yang, Y., Ge, Z., Zhang, M., Wei, L., Ma, M., Ma, Y., Chen, Y., 2018. Data on high performance supercapacitors based on mesoporous activated carbon materials with ultrahigh mesopore volume and effective specific surface area. Data Br. 18, 1448-1456. https://doi.org/10.1016/j. dib.2018.04.057.

10. Pang, L., Zou, B., Han, X., Cao, L., Wang, W., \& Guo, Y. (2016). One-step synthesis of high-performance porous carbon from corn starch for supercapacitor. Materials Letters, 184, 88-91.

11. Balathanigaimani, M.; Shim, W.-G.; Lee, M.-J.; Kim, C.; Lee, J.-W.; Moon, H., Highly porous electrodes from novel corn grains-based activated carbons for electrical double layer capacitors. Electrochem Commun 2008, 10 (6), 868-871.

12. Zhu, H.; Wang, X.; Yang, F.; Yang, X., Promising carbons for supercapacitors derived from fungi. Adv Mater 2011, 23 (24), 2745-2748.

13. Chen, W.; Zhang, H.; Huang, Y.; Wang, W., A fish scale based hierarchical lamellar porous carbon material obtained using a natural template for high performance electrochemical capacitors. J Mater Chem 2010, 20 (23), 4773-4775.

14. Raymundo-Piñero, E.; Cadek, M.; Béguin, F., Tuning carbon materials for supercapacitors by direct pyrolysis of seaweeds. Adv Funct Mater 2009, 19 (7), $1032-$ 1039.

15. Peng, C.; Yan, X.-b.; Wang, R.-t.; Lang, J.-w.; Ou, Y.-j.; Xue, Q.-j., Promising activated carbons derived from waste tea-leaves and their application in high performance supercapacitors electrodes. Electrochimica Acta 2013, 87, 401-408.

16. Kalpana, D.; Cho, S.; Lee, S.; Lee, Y.; Misra, R.; Renganathan, N., Recycled waste paper-A new source of raw material for electric double-layer capacitors. J Power Sources 2009, 190 (2), 587-591.

17. Lv, Y.; Gan, L.; Liu, M.; Xiong, W.; Xu, Z.; Zhu, D.; Wright, D. S., A self-template synthesis of hierarchical porous carbon foams based on banana peel for supercapacitor electrodes. J Power Sources 2012, 209, 152-157.

18. Li, X.; Xing, W.; Zhuo, S.; Zhou, J.; Li, F.; Qiao, S.-Z.; Lu, G.-Q., Preparation of capacitor's electrode from sunflower seed shell. Bioresource technology 2011, 102 (2), $1118-1123$.

19. Li, X.; Han, C.; Chen, X.; Shi, C., Preparation and performance of straw based activated carbon for supercapacitor in non-aqueous electrolytes. Microporous and Mesoporous Materials 2010, 131 (1), 303-309. 
20. Yun, Y. S.; Cho, S. Y.; Shim, J.; Kim, B. H.; Chang, S. J.; Baek, S. J.; Huh, Y. S.; Tak, Y.; Park, Y. W.; Park, S., Microporous carbon nanoplates from regenerated silk proteins for supercapacitors. Adv Mater 2013, 25 (14), 1993-1998.

21. Zhang, L.; Zhang, F.; Yang, X.; Leng, K.; Huang, Y.; Chen, Y., High-Performance Supercapacitor Electrode Materials Prepared from Various Pollens. Small 2013, 9 (8), 1342-1347.

22. Kuratani, K.; Okuno, K.; Iwaki, T.; Kato, M.; Takeichi, N.; Miyuki, T.; Awazu, T.; Majima, M.; Sakai, T., Converting rice husk activated carbon into active material for capacitor using three-dimensional porous current collector. J Power Sources 2011, 196 (24), 10788-10790.

23. Huang, W.; Zhang, H.; Huang, Y.; Wang, W.; Wei, S., Hierarchical porous carbon obtained from animal bone and evaluation in electric double-layer capacitors. Carbon 2011, 49 (3), 838-843.

24. Olivares-Marín, M.; Fernández, J.; Lázaro, M. J.; Fernández-González, C.; MacíasGarcía, A.; Gómez-Serrano, V.; Stoeckli, F.; Centeno, T. A., Cherry stones as precursor of activated carbons for supercapacitors. Mater Chem Phys 2009, 114 (1), 323-327.

25. Wu, F.-C.; Tseng, R.-L.; Hu, C.-C.; Wang, C.-C., Effects of pore structure and electrolyte on the capacitive characteristics of steam-and $\mathrm{KOH}$-activated carbons for supercapacitors. J Power Sources 2005, 144 (1), 302-309.

26. Sun, L.; Tian, C.; Li, M.; Meng, X.; Wang, L.; Wang, R.; Yin, J.; Fu, H., From coconut shell to porous graphene-like nanosheets for high-power supercapacitors. J Mater Chem A 2013, 1 (21), 6462-6470.

27. Zhang, W. L.; Zhao, M. Z.; Liu, R. Y.; Wang, X. F.; Lin, H. B., Hierarchical porous carbon derived from lignin for high performance supercapacitor. Colloid Surface A 2015, 484, 518-527.

28. Wang, K. L.; Cao, Y. H.; Wang, X. M.; Castro, M. A.; Luo, B.; Gu, Z. R.; Liu, J.; Hoefelmeyer, J. D.; Fan, Q. H., Rod-shape porous carbon derived from aniline modified lignin for symmetric supercapacitors. J Power Sources 2016, 307, 462-467.

29. Hu, S. X.; Zhang, S. L.; Pan, N.; Hsieh, Y. L., High energy density supercapacitors from lignin derived submicron activated carbon fibers in aqueous electrolytes. J Power Sources 2014, 270, 106-112.

30. Demir, M. (2017). Renewable Carbon from Lignin Biomass and Its Electrode and Catalyst Applications in Batteries, Supercapacitors, and Fuel Cells (Doctoral dissertation, Virginia Commonwealth University). 